\title{
Suberoylanilide hydroxamic acid: a potential epigenetic therapeutic agent for lung fibrosis?
}

\author{
Z. Wang*, C. Chen*, S.N. Finger", S. d/o Kwajah M.M" ${ }^{\#}$, M. Jung", H. Schwarz", \\ N. Swanson ${ }^{+}$, R.R. Lareu ${ }^{\star,+, \$}$ and M. Raghunath*,
}

ABSTRACT: Pulmonary fibrosis represents a fatal stage of interstitial lung diseases of known and idiopathic aetiology. No effective therapy is currently available. Based on an indication-discovery approach we present novel in vitro evidence that the histone deacetylases inhibitor suberoylanilide hydroxamic acid (SAHA), an FDA approved anti-cancer drug, has antifibrotic and anti-inflammatory potential.

Human lung fibroblasts (fetal, adult and idiopathic adult pulmonary fibrosis) were treated with transforming growth factor (TGF)- $\beta 1$ with or without SAHA. Collagen deposition, $\alpha$-smooth muscle actin ( $\alpha$-SMA) expression, matrix metalloproteinase (MMP)1 activity, tissue inhibitor of MMP (TIMP)1 production, apoptosis and cell proliferation were assessed. Pro-inflammatory cytokines relevant to pulmonary fibrosis were assayed in SAHA-treated human peripheral blood mononuclear cells (PBMC) and its subpopulations.

SAHA abrogated TGF- $\beta 1$ effects on all the fibroblast lines by preventing their transdifferentiation into $\alpha$-SMA positive myofibroblasts and increased collagen deposition without inducing apoptosis. However, MMP1 activity and TIMP1 production was modulated without a clear fibrolytic effect. SAHA also inhibited serum-induced proliferation of the fibroblast lines and caused hyperacetylation of $\alpha$-tubulin and histone. Cytokine secretion was inhibited from PBMC and lymphocytes at nonapoptotic concentrations.

Taken together, these data demonstrate combined antifibrotic and anti-inflammatory properties of SAHA, suggesting its therapeutic potential for pulmonary fibrosis.

KEYWORDS: Antifibrotic, collagen, fibroblast, histone deacetylase inhibitor, pulmonary fibrosis, suberoylanilide hydroxamic acid

nterstitial lung diseases constitute a diverse set of lung disorders with different levels of inflammation and fibrosis resulting in irreversible loss of lung function and ultimately respiratory failure [1]. The most common representative of interstitial lung diseases is idiopathic pulmonary fibrosis (IPF). The general pathological feature of IPF is the excessive deposition of collagen I which destroys the architecture of the normal lung parenchyma [2]. Patients with IPF have a median survival of 2-3 yrs after diagnosis [1]. The current therapy for IPF is based on reducing inflammation with corticosteroids and immunosuppressive drugs. However, the apparent ineffectiveness of this regimen has led to doubts about the initial role of inflammation in the pathogenesis of IPF [3, 4] and to the concept that correcting the activated state of IPF fibroblasts/myofibroblasts [5] might be more important than an anti-inflammatory strategy. New therapeutic strategies, including antioxidant agents, anti-endothelial cell antibody, anti-cytokine therapy and antifibrotic agents, such as pirfenidone, have been evaluated in clinical trials without showing significant improvement in the treatment of IPF [6]. Clearly, IPF remains a large therapeutic challenge and the development of effective antifibrotic drugs is urgently required.

To explore new antifibrotic drugs, we have been investigating the therapeutic potential of histone deacetylase inhibitors (HDACi). HDACi are small organic molecules which change gene expression profiles at the epigenetic level and change protein function by inhibiting the activity of histone deacetylases. HDACi are of prime interest in cancer research because they induce apoptosis and cell cycle arrest in malignant cells and are anti-angiogenic [7]. Recently published studies suggested that HDACi might also inhibit

\section{AFFILIATIONS}

*Tissue Modulation Laboratory, Division of Bioengineering, Faculty of Engineering,

"Dept of Physiology, National University of Singapore,

${ }^{\S}$ NUS Tissue Engineering Program, Dept of Orthopedic Surgery, fDept of Biochemistry, Yong Loo Lin School of Medicine, National University of Singapore, Singapore, "Institute of Pharmaceutical Sciences, University of Freiburg Freiburg, Germany, and

${ }^{+}$Molecular Hepatology, School of Medicine and Pharmacology, Faculty of Medicine, Dentistry and Heath Sciences, The University of Western Australia, Perth, Australia.

CORRESPONDENCE

M. Raghunath

Division of Bioengineering, Faculty of Engineering, and Dept of Biochemistry

Yong Loo Lin School of Medicine Division Office Block E3A \#04-15

7 Engineering Dr. 1

Singapore 117574

E-mail: bierm@nus.edu.sg

Received:

June 052008

Accepted after revision:

Jan 252009

First published online Feb 122009 
collagen production in different fibroblast types. Phenylbutyrate decreased basal levels and transforming growth factor (TGF)- $\beta 1$-stimulated $\alpha 1$ (I) collagen messenger RNA (mRNA) and protein levels in the lung fetal fibroblast IMR-90 [8]. Trichostatin A (TSA) was reported to inhibit synthesis of collagen type I and III and $\alpha$-smooth muscle actin ( $\alpha$-SMA) both at the protein and mRNA level in primary cultured rat hepatic stellate cells [9], and in TGF- $\beta 1$-stimulated rat skin fibroblasts [10]. In addition, collagen production from systemic sclerosis fibroblasts was inhibited and total collagen deposition was reduced in bleomycin-induced skin fibrosis in mice by TSA [11].

Based on an indication-discovery approach, we evaluated suberoylanilide hydroxamic acid (SAHA) (Zolina ${ }^{\circledR}$ (vorinostat), an FDA-approved HDACi, which is already in clinical use as a third-line drug for the treatment of cutaneous T-cell lymphoma and under evaluation for other types of cancer [12]. Herein, we studied the antifibrotic potential of SAHA in TGF- $\beta 1$-treated fetal lung fibroblasts (FLF), adult lung fibroblasts (ALF) and IPF lung fibroblast (ILF) lines, and its potential anti-inflammatory effect in peripheral blood mononuclear cells (PBMC), lymphocytes and peripheral monocytes.

\section{MATERIALS AND METHODS Compounds and reagents}

SAHA was synthesised as described previously [13], dissolved in dimethyl sulfoxide to $50 \mathrm{mM}$ and stored at $-80^{\circ} \mathrm{C}$. All reagents were from Sigma Aldrich (St. Louis, MI, USA) unless stated otherwise.

\section{Treatment of lung fibroblast lines}

Normal human FLF IMR-90 (CCL-186; ATCC, Manassas, VA, USA), ALF CCD-19 Lu (CCL-210; ATCC) and ILF LL29 (CCL134; ATCC) were cultured in 10\% fetal bovine serum (FBS) Dulbecco's modified Eagle medium (GIBCO Invitrogen, Singapore). Cells, except for proliferation assays, were seeded at $1 \times 10^{4} \cdot$ well $^{-1}, 5 \times 10^{4} \cdot$ well $^{-1}$ and $2.5 \times 10^{5} \cdot$ well $^{-1}$ in 96-, 24and 6-well plates, respectively. After $24 \mathrm{~h}$, fibroblasts were treated with different concentrations of SAHA with or without TGF- $\beta 1$ (5 ng. $\mathrm{mL}^{-1}$; R\&D Systems, Minneapolis, MN, USA) in serum-free Dulbecco's modified Eagle medium plus $30 \mu \mathrm{g} \cdot \mathrm{mL}^{-1}$ ascorbic acid phosphate (Wako Pure Chemicals Industries Ltd, Osaka, Japan) in the presence of $100 \mu \mathrm{g} \cdot \mathrm{mL}^{-1}$ dextran sulfate 500 kDa (pK Chemicals A/S, Koge, Denmark), for the rapid deposition of collagen I within $24 \mathrm{~h}$ [14].

\section{Quantitative immunocytochemistry}

Fibroblasts cultured in 96-well Lumox plates with an optical bottom (Greiner BioOne, Gottingen, Germany) were fixed with absolute methanol and air dried for $15 \mathrm{~min}$. Cell layer was blocked with $3 \%$ bovine serum albumin in PBS for $30 \mathrm{~min}$ followed by incubation with primary antibodies for $90 \mathrm{~min}$. Secondary antibodies and nuclear stain 4,6-diamidino-2phenylindole (DAPI; Invitrogen Molecular Probes, Carlsbad, CA, USA) were stained for $30 \mathrm{~min}$. The cell layer was washed with PBS three times after each step. Antibodies against collagen type I (1:500), $\alpha$-SMA (1:100; Dako, Glostrup, Denmark) or acetylated $\alpha$-tubulin (1:500) were from mouse and acetylated histone 3 (1:200; Upstate Biotechnology, New York, NY, USA) from rabbit. Secondary antibodies were Alexa Fluor (AF) 594 goat anti-mouse and AF488 goat anti-rabbit (both 1:400; Invitrogen Molecular Probes). The fluorescence intensity was quantified using the PHERAstar microplate reader with a focusing lens system (BMG Labtech, Mornington, Australia). The ratio of the fluorescence intensity of the AF dye to DAPI was used to normalise the relative amount of the antigen to cell number. Images were taken with an Olympus LX71 epifluorescence microscope (Olympus, Tokyo, Japan).

\section{Biochemical assay of collagen matrix}

Deposited collagen was extracted from cell cultures and analysed as described previously [14]. Briefly, cell layers in 24-well plates were digested in situ with $250 \mu \mathrm{g} \cdot \mathrm{mL}^{-1}$ porcine gastric mucosa pepsin (Roche, Basel, Switzerland) in $0.1 \mathrm{M}$ $\mathrm{HCl}$ for $2 \mathrm{~h}$ and neutralised with $1 \mathrm{M} \mathrm{NaOH}$. Extracts were visualised using SDS-PAGE gels with silver staining and analysed by densitometry.

\section{Western blot}

Western blots were performed as previously described [14]. Briefly, proteins were extracted with loading buffer $(50 \mathrm{mM}$ Tris-Cl pH 6.8, 2\% SDS, $0.1 \%$ bromophenol blue and $10 \%$ glycerol) with $5 \mathrm{mM}$ dithiothreitol and separated on $10 \%$ SDSPAGE gels. Primary antibodies against acetylated $\alpha$-tubulin (1:2500), $\alpha$-SMA (1:500) and $\beta$-actin (1:1000) were from mouse, and acetylated histone 3 (1:3000; Upstate Biotechnology) from rabbit. Secondary antibodies were goat anti-mouse and goat anti-rabbit horse radish peroxidase (both 1:1000; Pierce, Rockford, IL, UK). Blots were developed with Amersham ${ }^{\mathrm{TM}}$ ECL plus Western blotting detection system (GE Healthcare, Chalfont St Giles, UK) and chemiluminescence was captured with a VersaDoc Imaging System model 5000 (Bio-Rad, Hercules, CA, USA).

\section{Flow cytometry analysis of $\alpha$-SMA expression}

Fibroblasts in suspension were fixed with $2 \%$ paraformaldehyde in PBS and permeabilised with $0.1 \%$ saponin in PBS for $15 \mathrm{~min}$. The suspension was incubated with mouse anti- $\alpha$ SMA (1:1000) and AF488 chicken anti-mouse (1:100; Invitrogen Molecular Probes), each for $30 \mathrm{~min}$ at $4{ }^{\circ} \mathrm{C}$. The cells were washed with PBS twice after each step. AF488 was detected by flow cytometry.

\section{Adherent cytometry}

For proliferation assay, cells were seeded at $2 \times 10^{4} \cdot$ well $^{-1}$ in 24 well plate and cultured in 10\% FBS in the presence or absence of $5 \mu \mathrm{M}$ SAHA. After 3 days, fibroblasts were stained with DAPI after methanol fixation. Nine image sites covering $71 \%$ of the total well area were acquired at $2 \times$ magnification using a Nikon TE600 fluorescence microscope with an automated Ludl stage (BioPrecision 2; Ludl Electronic Products Ltd, Hawthorne, NY, USA) and analysed using the Metamorph ${ }^{\circledR}$ Imaging System software (Molecular Devices, Downingtown, PA, USA). A nucleus was defined as a fluorescent region with a length of $10-15 \mu \mathrm{m}$ and a pixel intensity value of 10 units above background.

\section{Carboxyfluoroscein succinimidyl ester labelling and detection}

Fibroblasts at $10^{6} \cdot \mathrm{mL}^{-1}$ were pre-labelled with $2 \mu \mathrm{M}$ carboxyfluoroscein succinimidyl ester (CFSE; Invitrogen Molecular 

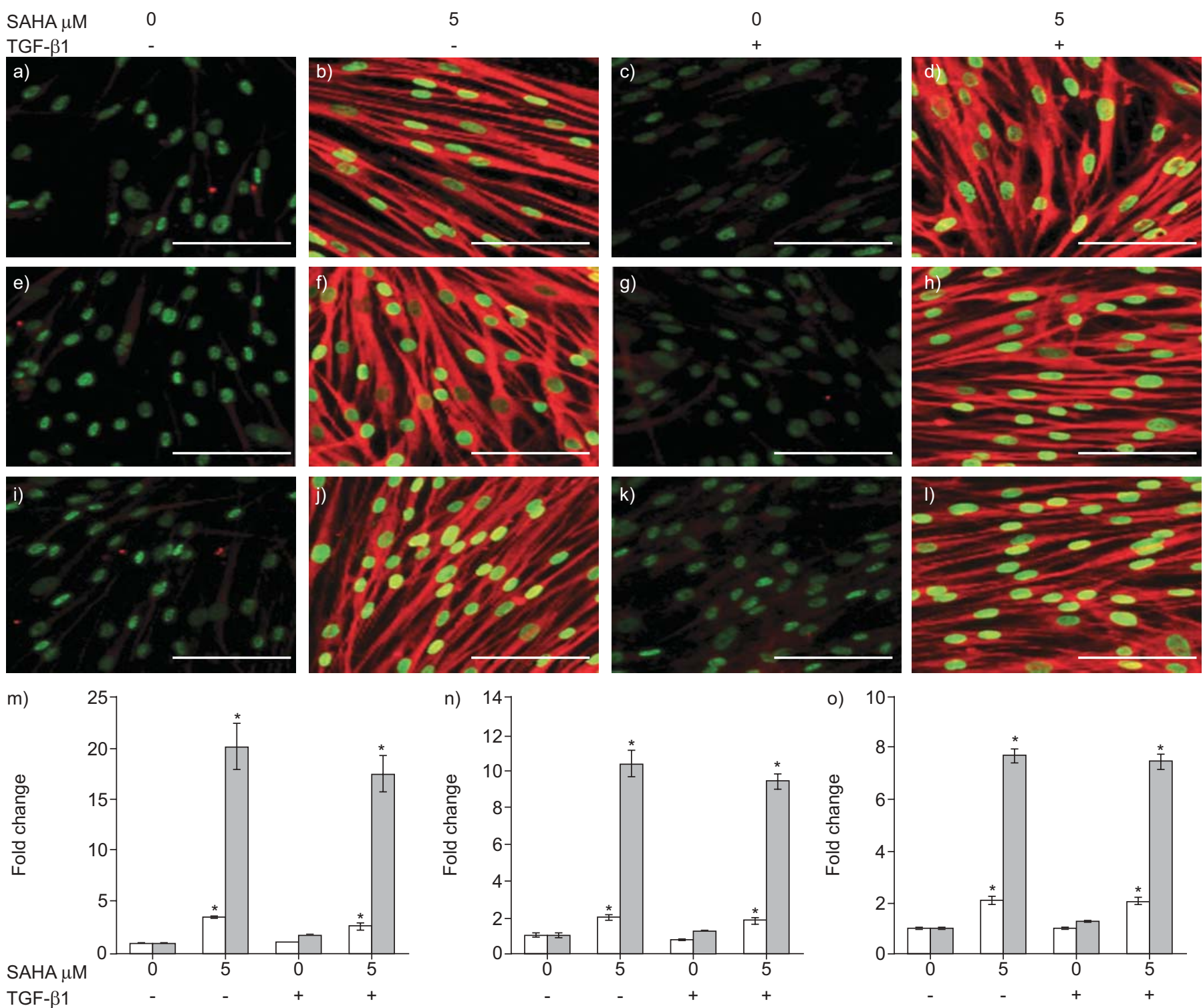

о) 10

TGF- $\beta 1$

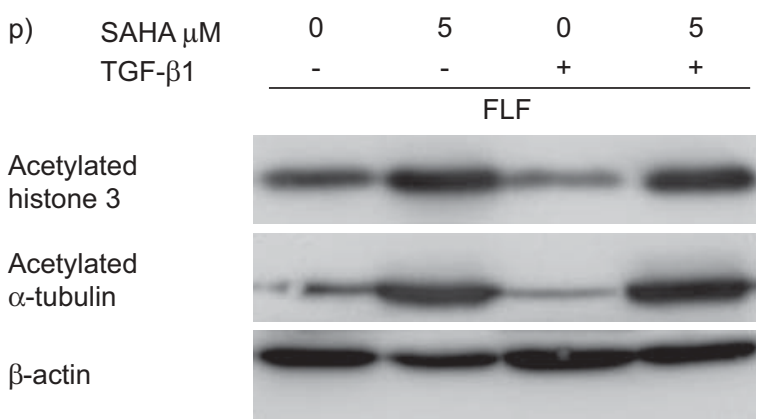

\begin{tabular}{rrrr}
0 & 5 & 0 & 5 \\
- & - & + & + \\
\hline \multicolumn{4}{c}{ ALF }
\end{tabular}
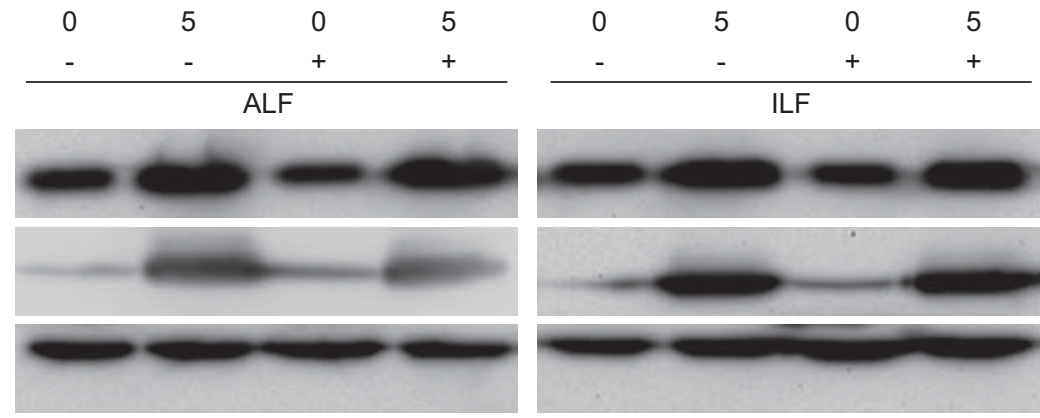

FIGURE 1. Suberoylanilide hydroxamic acid (SAHA) induces hyperacetylation of histone and $\alpha$-tubulin. Fetal lung fibroblasts (FLF), adult lung fibroblasts (ALF) and idiopathic pulmonary fibrosis lung fibroblasts (ILF) were treated with or without transforming growth factor (TGF)- $\beta 1$ ( $5 \mathrm{ng} \cdot \mathrm{mL}^{-1}$ ) and $5 \mu \mathrm{M} \mathrm{SAHA}$ for $24 \mathrm{~h}$. The acetylation of histone 3 and $\alpha$-tubulin were quantified by quantitative immunocytochemistry (QICC) and Western blot. a, b) Immunocytochemistry of acetylation of histone 3 (green) and $\alpha$ tubulin (red) in a-d) FLF (IMR-90), e-h) ALF (CCD-19 Lu) and i-l) ILF (LL29). Scale bars=50 $\mu \mathrm{m}$. m-0) Average fold change of acetylation of histone 3 ( $\square$ ) and $\alpha$-tubulin ( $\square$ ) from quadruplicates were quantified by QICC. m) FLF, n) ALF and o) ILF. *: p $<0.05$ compared with untreated control. p) Western blots of acetylated histone 3 and acetylated $\alpha$-tubulin. 
Probe). With or without $5 \mu \mathrm{M}$ SAHA, $1 \times 10^{5}$ cells $\cdot$ well $^{-1}$ were seeded into 6-well plate and cultured in 10\% FBS. After 3 days, fibroblasts were trypsinised, washed twice with PBS and CFSE was detected by flow cytometry.

\section{Matrix metalloproteinase 1 activity and tissue inhibitor of matrix metalloproteinase 1 ELISA}

Fibroblasts were treated with or without TGF- $\beta 1\left(5 \mathrm{ng} \cdot \mathrm{mL}^{-1}\right)$ and SAHA at $5 \mu \mathrm{M}$ for $24 \mathrm{~h}$. Matrix metalloproteinase (MMP)1 activity and tissue inhibitor of MMP (TIMP) 1 production were determined using the Fluorokine E kit and the Quantikine human TIMP1 kit (both R\&D Systems) according to the manufacturers protocol. 4-aminophenylmercuric acetate (APMA)-activated MMP1 was detected by cleavage of fluorescence-labelled substrate peptide. Concentrations of active MMP1 and TIMP1 were calculated from standard curves obtained from standards run in parallel.

\section{Apoptosis assay}

Briefly, $10^{6}$ fibroblasts, PBMC, lymphocytes or monocytes were stained with $100 \mu \mathrm{L}$ fluorescein isothiocyanate-annexin $\mathrm{V}$ and propidium iodide (PI) labelling solution for $15 \mathrm{~min}$ at room

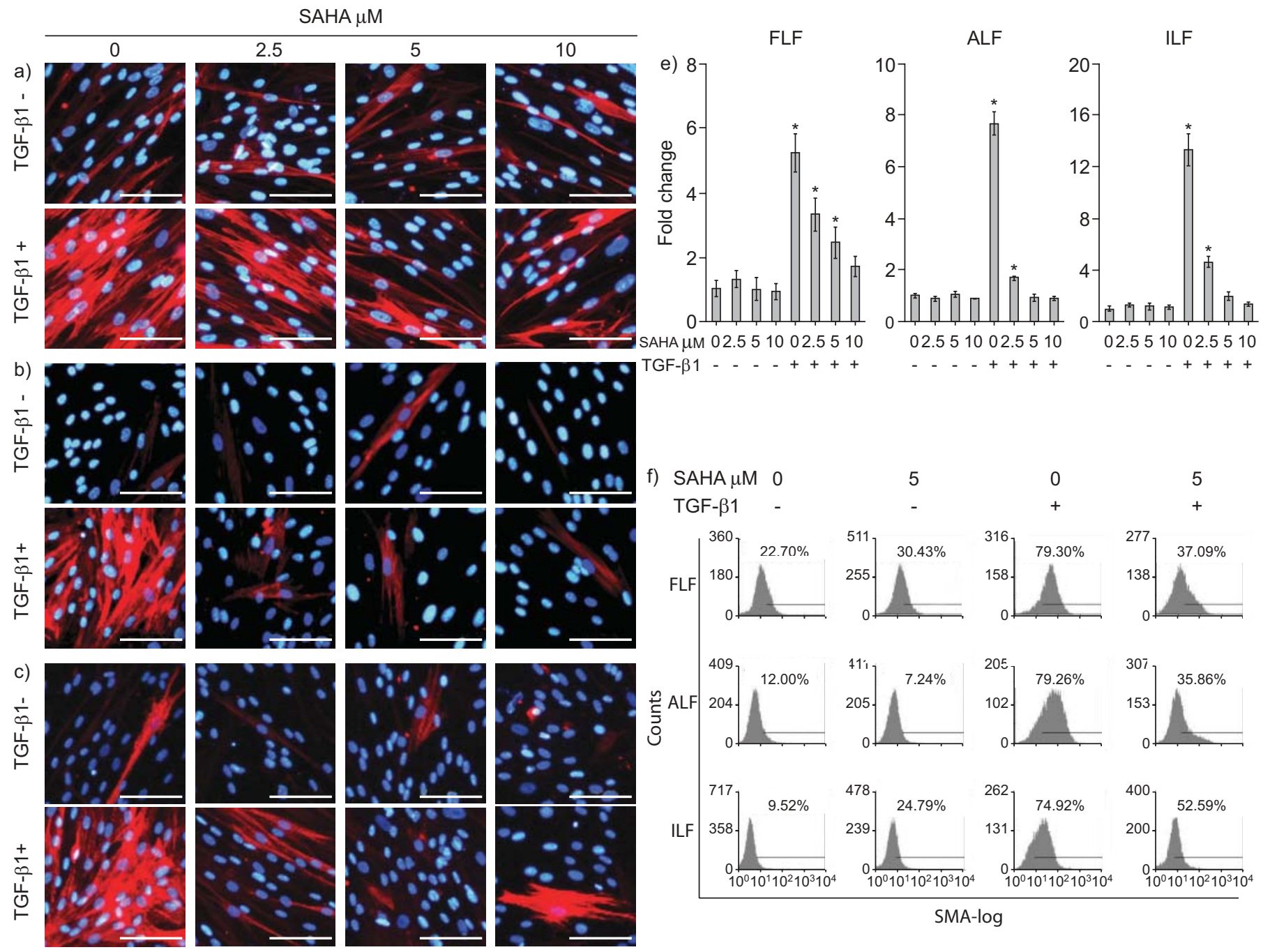

\begin{tabular}{|c|c|c|c|c|c|c|c|c|c|c|c|c|}
\hline d) SAHA $\mu M$ & 0 & 5 & 0 & 5 & 0 & 5 & 0 & 5 & 0 & 5 & 0 & 5 \\
\hline TGF- $\beta 1$ & - & - & + & + & - & - & + & + & - & - & + & + \\
\hline & \multicolumn{4}{|c|}{ FLF } & \multicolumn{4}{|c|}{ ALF } & \multicolumn{4}{|c|}{ ILF } \\
\hline$\alpha$-SMA & - & & & - & & & - & & & & & \\
\hline
\end{tabular}

FIGURE 2. Suberoylanilide hydroxamic acid (SAHA) inhibits transdifferentiation of fibroblasts into myofibroblasts. a) Fetal lung fibroblasts (FLF), b) adult lung fibroblasts (ALF) and c) idiopathic pulmonary fibrosis lung fibroblasts (ILF) were treated with or without transforming growth factor (TGF) $-\beta 1$ ( $\left.5 \mathrm{ng} \cdot \mathrm{mL}^{-1}\right)$ and increasing concentrations of SAHA $(2.5,5$ and $10 \mu \mathrm{M})$ for $24 \mathrm{~h}$. The expression of $\alpha$-smooth muscle actin ( $\alpha$-SMA) was evaluated by quantitative immunocytochemistry (QICC) and Western blot. a-c) Representative immunocytochemistry images ( $\alpha$-SMA, red; DAPI, blue). Scale bars $=50 \mu \mathrm{m}$. d) Western blots of $\alpha$-SMA. e) Average $\alpha$-SMA fold changes from quadruplicates were quantified by QICC. *: $p<0.05$ compared with untreated control. f) Flow cytometry analysis of the percentage of $\alpha$-SMA positive cells. 


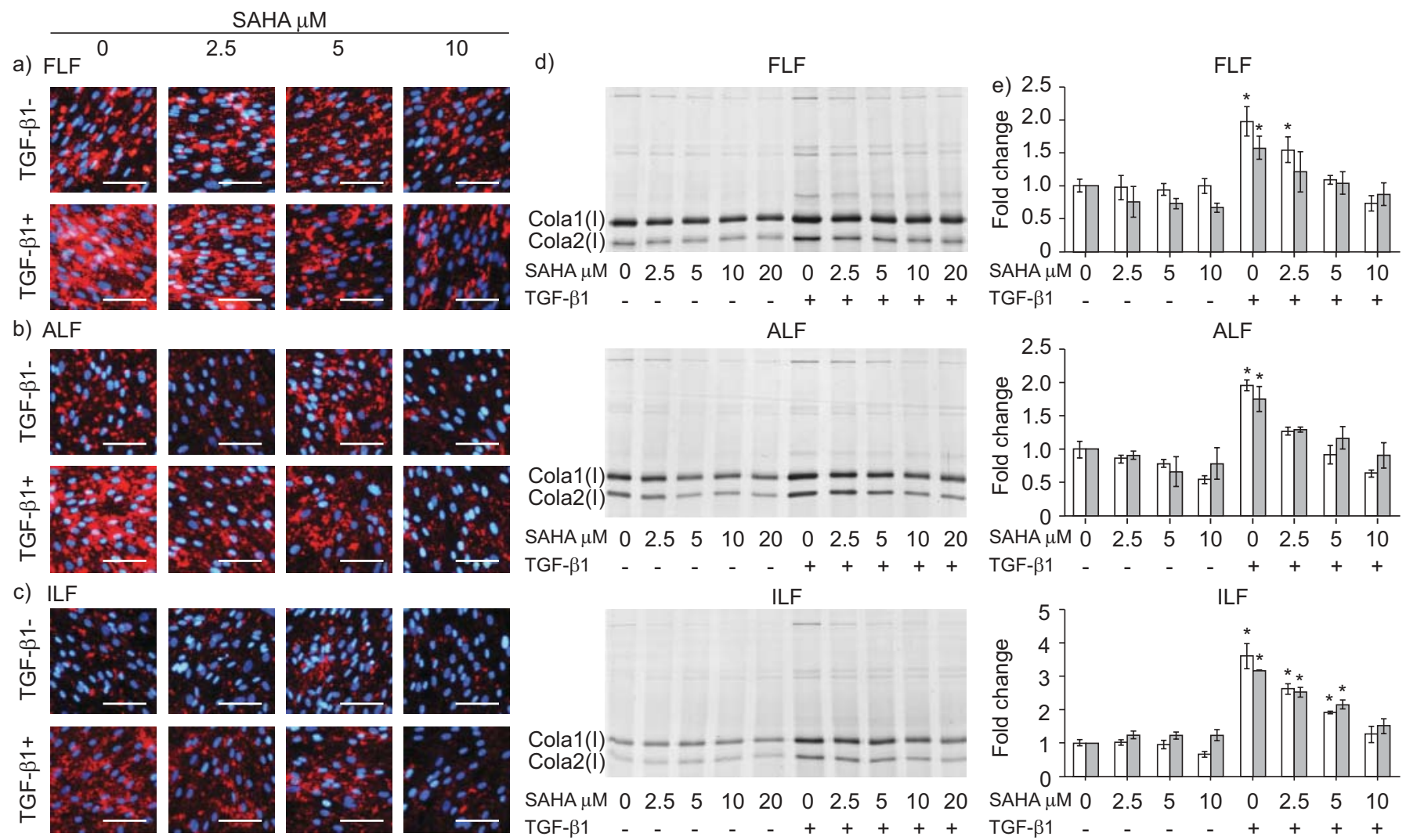

FIGURE 3. Suberoylanilide hydroxamic acid (SAHA) inhibits transforming growth factor (TGF)- $\beta 1$-induced collagen production. Fetal lung fibroblasts (FLF), adult lung fibroblasts (ALF) and idiopathic pulmonary fibrosis lung fibroblasts (ILF) were treated with or without TGF- $\beta 1\left(5 \mathrm{ng} \cdot \mathrm{mL}^{-1}\right)$ and increasing concentrations of SAHA (2.5, 5,10 and $20 \mu \mathrm{M}$ ) for $24 \mathrm{~h}$. The expression of collagen I was quantified by quantitative immunocytochemistry (QICC) and SDS-PAGE. a-c) Representative immunocytochemistry (collagen I, red; DAPI, blue). Scale bars $=50 \mu \mathrm{m}$. d) Representative SDS-PAGE from three independent experiments. e) Average collagen fold changes were quantified from quadruplicates by QICC and three independent SDS-PAGE gels by biochemical assay. Statistically significant differences in collagen deposition quantified by QICC ( $\square$ ) and biochemical assay $(\square){ }^{*}$ : $p<0.05$ compared with untreated control.

temperature using Annexin-V-FLUOS staining kit (Roche, Penzberg, Germany) according to the manufacturers protocol. The percentages of annexin- $\mathrm{V}$ single positive, annexin-V/PI double-positive and annexin-V/PI double-negative cells were determined by flow cytometry.

\section{Treatment of PBMC and its subpopulations and cytokines ELISA}

Human PBMC were isolated from buffy coats using Histopaque-1077 (Sigma Aldrich) from blood of healthy volunteers at the National University Hospital (Singapore) under informed consent and local international review board (National University Hospital) approval. Monocytes were further isolated from PBMC with the human Monocyte Isolation Kit II (Miltenyi Biotec, Bergisch Gladbach, Germany). Lymphocytes were isolated as the cells remained on the magnetic column after removal of monocytes. PBMCs, lympocytes and monocytes were seeded at $1 \times 10^{6} \cdot \mathrm{mL}^{-1}$ in $10 \%$ FBS RPMI-1640 and incubated with $0.5 \mathrm{ng} \cdot \mathrm{mL}^{-1}$ phorbol myristate acetate plus $50 \mathrm{nM}$ calcium ionophore A23187 (PMA/CI) alone or with 0.5 or $1 \mu \mathrm{M}$ SAHA for 24 or $48 \mathrm{~h}$. Supernatants were collected and frozen at $-20^{\circ} \mathrm{C}$. The cytokines assayed were tumour necrosis factor (TNF)- $\alpha$, interleukin (IL)-13, IL-10
(eBioscience, San Diego, CA, USA), IL-6, IL-8 and TGF- $\beta 1$ (R\&D Systems) following the manufacturers protocol.

\section{Statistical analysis}

Statistical analysis was performed using SPSS 12.0. (SPSS Inc., Chicago, IL, USA) Equality of error variances was tested by Levene's test. Data with equal variances were compared by Turkey test and data with unequal variances by Dunnet's T3 test. Probability values of $\mathrm{p}<0.05$ were accepted as the level of statistical significance.

\section{RESULTS}

SAHA induces hyperacetylation of histone 3 and $\alpha$-tubulin Basal acetylation levels of histone and $\alpha$-tubulin were noted in untreated FLF, ALF and ILF, but were significantly increased in the presence of SAHA after $24 \mathrm{~h}$ as assessed by quantitative immunocytochemistry (QICC; fig. 1a-o) and Western blot (fig. 1p), indicating that SAHA inhibited HDACs activity. TGF- $\beta 1$ did not change the acetylation of histone 3 and $\alpha$ tubulin significantly in the presence or absence of SAHA.

\section{SAHA inhibits TG $\beta$-1-induced myofibroblast transdifferentiation}

Untreated cell cultures of all the fibroblasts lines showed low levels of $\alpha$-SMA expression by immunocytochemistry (fig. 2a-c), 

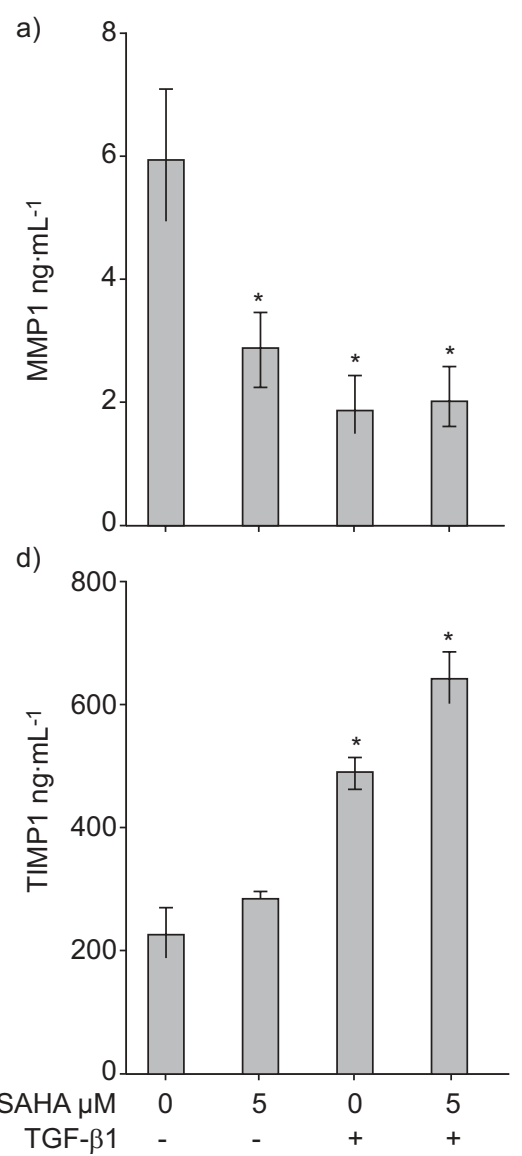
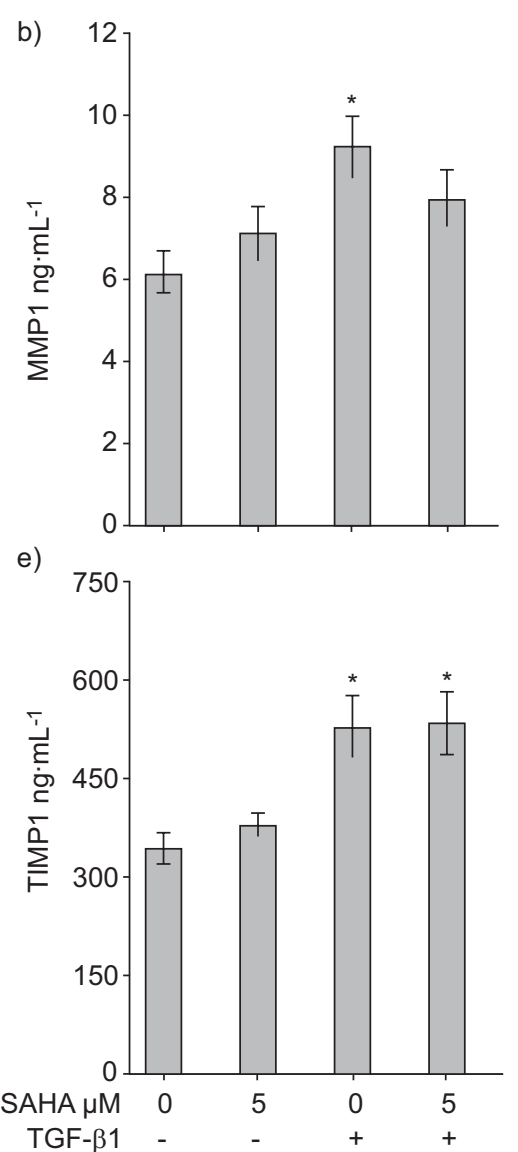
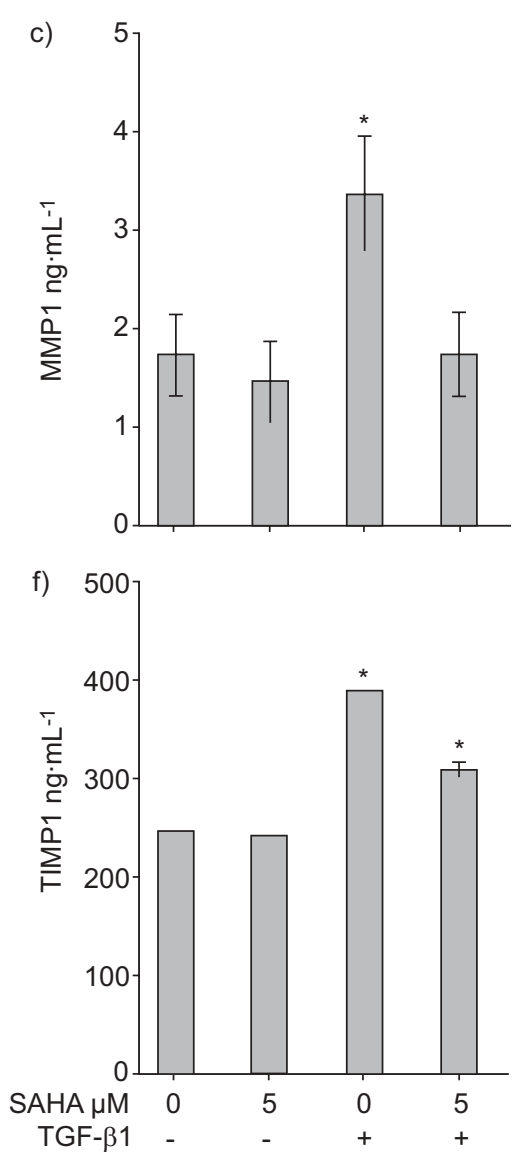

FIGURE 4. Suberoylanilide hydroxamic acid (SAHA) regulates matrix metalloproteinase (MMP) 1 and tissue inhibitor of MMP (TIMP) 1 differentially in tested fibroblasts. a, d) Fetal lung fibroblasts (FLF), b, e) adult lung fibroblasts (ALF), and c, f) idiopathic pulmonary fibrosis lung fibroblasts (ILF) were treated with or without transforming growth factor (TGF)- $\beta 1$ ( $5 \mathrm{ng} \cdot \mathrm{mL}^{-1}$ ) and SAHA at $5 \mu \mathrm{M}$ for $24 \mathrm{~h}$. Media were collected and analysed for 4-aminophenylmercuric acetate (APMA)-active MMP1 activity and TIMP1 by ELISA. The experiments were performed three times with three different batches of samples, and one representative result was used to show the averages of a-c) APMAactivated MMP1 and $d-f$ ) TIMP1 from duplicates of each condition. *: $p<0.05$ compared with untreated control.

Western blot (fig. 2d), and flow cytometry analyses (fig. 2f). Microscopy revealed that this was due to a few interspersed cells that were positive for $\alpha$-SMA. Although TGF- $\beta 1$ triggered transdifferentiation of the majority of fibroblasts into myofibroblasts in all three fibroblast lines, ILF were the most responsive with a 14 -fold enhanced $\alpha$-SMA expression followed by eightfold in ALF and five-fold in FLF, as assessed by QICC (fig. 2e). SAHA abolished this effect in a dose-dependent manner from 2.5 to $10 \mu \mathrm{M}$ by both reducing the expression of $\alpha$-SMA and percentage of $\alpha$-SMA positive fibroblasts (fig. 2).

\section{SAHA inhibits TG/-1-induced collagen production}

Both QICC (fig. 3a-c) and quantitative gel electrophoresis (fig. 3d) demonstrated increased collagen deposition in response to TGF- $\beta 1$. ILF were the most responsive with an increase of 3-4-fold in comparison with 1.5-2-fold in FLF and ALF, respectively (fig. 3e). SAHA abrogated TGF- $\beta 1$-induced collagen deposition dose-dependently in all the fibroblasts lines. At $10 \mu \mathrm{M}$, SAHA completely counteracted the effect of TGF- $\beta 1$ on ILF by limiting collagen deposition to pre-treatment levels, and $5 \mu \mathrm{M}$ on FLF and ALF. However, SAHA alone did not significantly affect the basal level of collagen deposition in the three fibroblast lines.

\section{SAHA regulates MMP1 and TIMP1 differentially in tested fibroblasts}

TGF- $\beta 1$ reduced the total potentially activated (APMAactivated) MMP1 in FLF to one-third, but doubled it in ALF and ILF. In FLF, $5 \mu \mathrm{M}$ SAHA had no effect on TGF- $\beta 1$-reduced MMP1, whereas it decreased MMP1 to the basal level in ALF and ILF. SAHA alone inhibited APMA-activated MMP1 in FLF by $\sim 50 \%$, but did not affect MMP1 in ALF and ILF (fig. $4 a-c$ ). TIMP1 was increased by TGF- $\beta 1$ in all three fibroblast lines to $2-2.5$-fold. It was further induced by SAHA only in FLF, but remained unchanged in ALF and was reduced slightly in ILF. SAHA alone did not change TIMP1 production significantly (fig. $4 \mathrm{~d}-\mathrm{f}$ ).

\section{SAHA is not pro-apoptotic in the tested fibroblasts}

Annexin V and PI staining showed that SAHA at $5 \mu \mathrm{M}$ did not induce apoptosis in all three fibroblast lines in the presence or absence of TGF- $\beta 1$ (fig. 5).

\section{SAHA inhibits serum-induced fibroblast proliferation}

Adherent cytometry revealed that $5 \mu \mathrm{M}$ SAHA inhibited proliferation of the three fibroblast lines in $10 \%$ FBS after the first day and stopped cell proliferation afterwards (fig. 6a-c). 


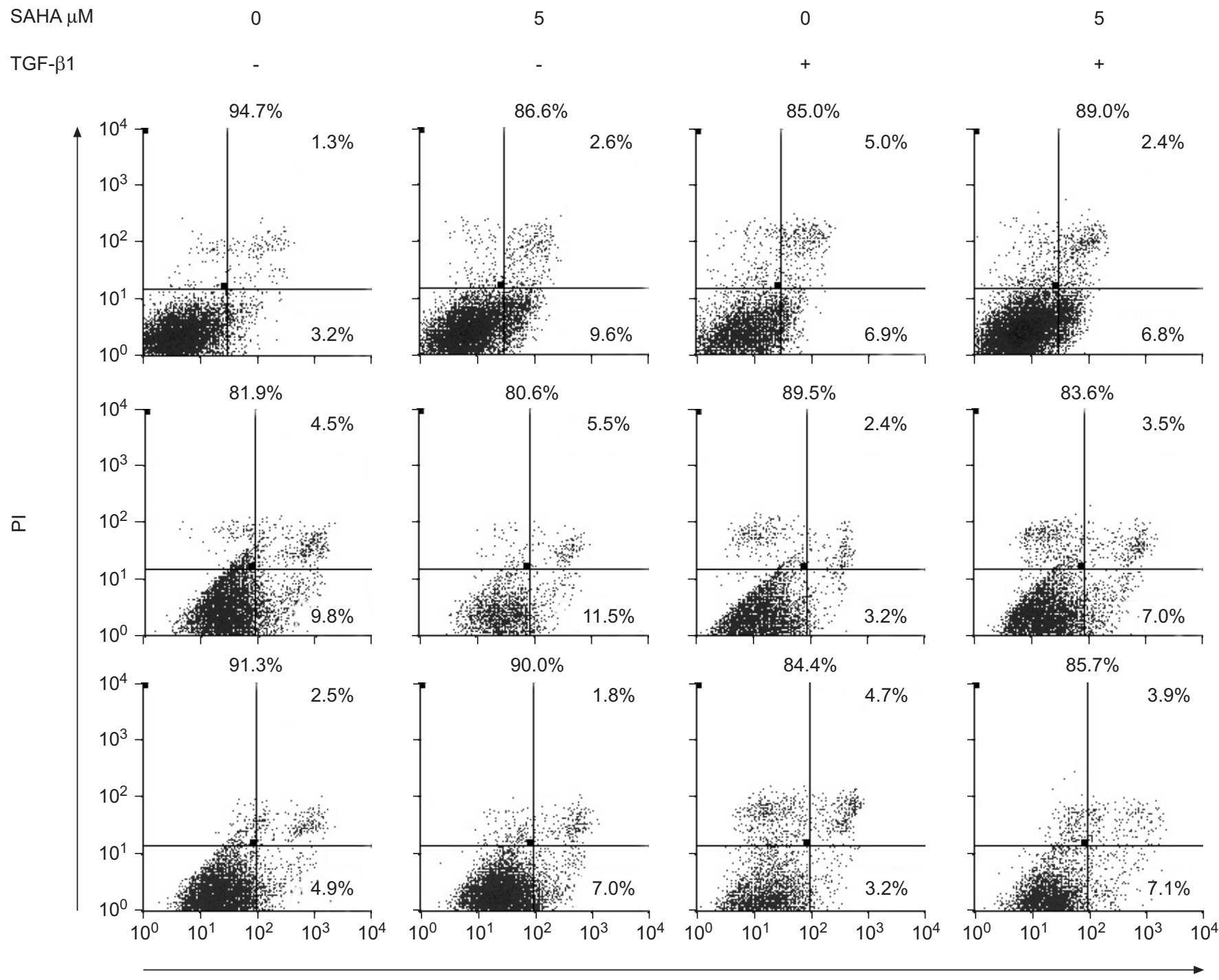

Annexin V

FIGURE 5. Suberoylanilide hydroxamic acid (SAHA) is not pro-apoptotic in fetal lung fibroblasts (top row), adult lung fibroblasts (middle row) and idiopathic pulmonary fibrosis lung fibroblasts (bottom row). Fibroblasts were treated with or without transforming growth factor (TGF)- $\beta 1$ ( $5 \mathrm{ng} \cdot \mathrm{mL}^{-1}$ ) and SAHA at $5 \mu \mathrm{M}$ for $24 \mathrm{~h}$. Cells were stained with annexin $\mathrm{V}$ and propidium iodide (PI). The percentage of apoptotic (annexin- $\mathrm{V}$ single positive) and necrotic (annexin-V/PI double-positive) cells are labelled at the corresponding regions of each graph, and that of living cells (annexin-V/PI double-negative) above each graph.

Higher fluorescence intensity of CFSE was detected in SAHAtreated fibroblasts suggesting reduced cell proliferation (fig. 6d).

\section{SAHA changes cytokine release in activated PBMC and its subpopulations}

SAHA at concentrations applied for fibroblast cultures induced apoptosis in PBMC and its subpopulations, and thus its antiinflammatory property was tested at lower concentrations (0.5 and $1 \mu \mathrm{M}$ ) without affecting cell viability (data not shown). In PBMC and lymphocytes stimulated with PMA/CI, secretion of TNF- $\alpha$, IL-8, IL-13 and IL-10 were decreased, while levels of TGF- $\beta 1$ remained unchanged. SAHA did not alter secretion of IL-6 in PBMC, but reduced it in lymphocytes. In monocytes under the same stimulus, SAHA decreased secretion of IL-10 without affecting the other tested cytokines (fig. 7, table 1).

\section{DISCUSSION}

In the past $15 \mathrm{yrs}$, the incidence and mortality of pulmonary fibrosis, particularly IPF, has increased significantly in patients aged $>50$ yrs [15]. No current treatment can effectively stop the pathological scarring process in the lung parenchyma. Several potential antifibrotic drugs are in different phases of clinical trials, but a clear benefit is still not in sight [6] Therefore, we have explored new indications for drugs already in clinical use, which could potentially broaden and accelerate the therapeutic options for pulmonary fibrosis.

We have shown that the anti-cancer drug SAHA limits the profibrotic response induced by TGF- $\beta 1$ in ILF, as well as FLF and ALF, without inducing apoptosis. This was demonstrated by the restoration of collagen I deposition to normal levels and suppression of $\alpha$-SMA expression, indicating that the 


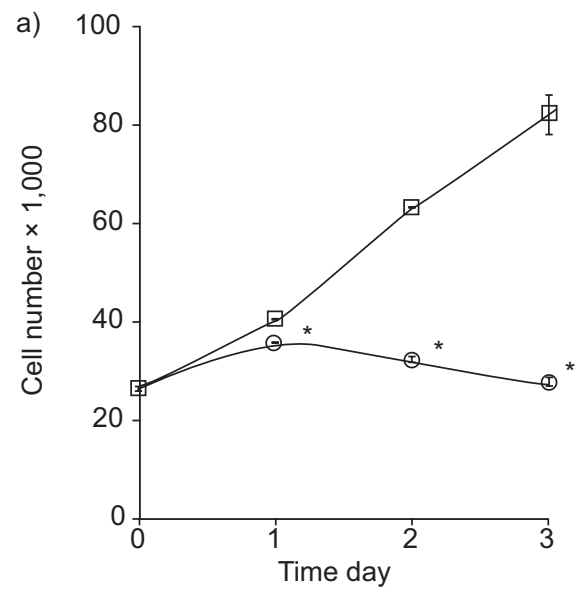

b)

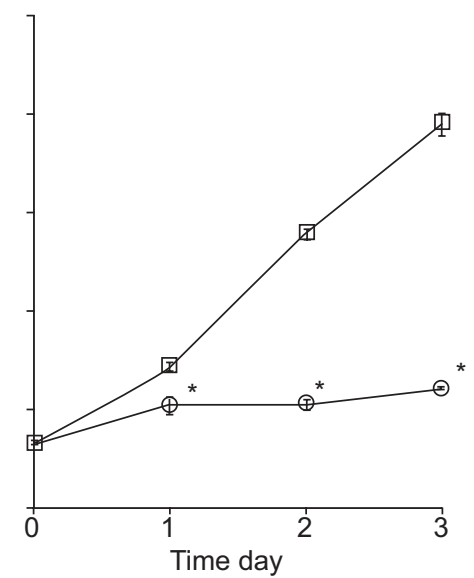

d)

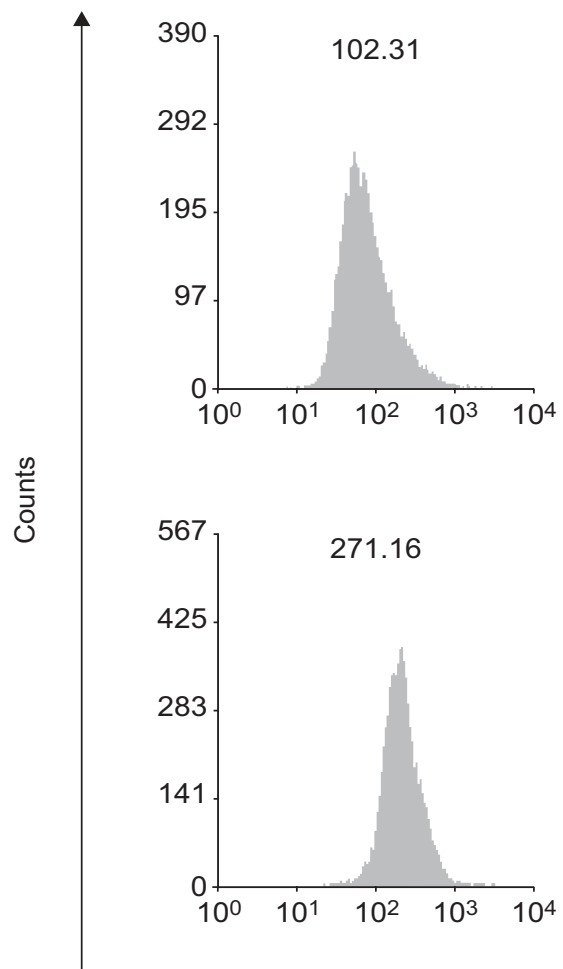

c)
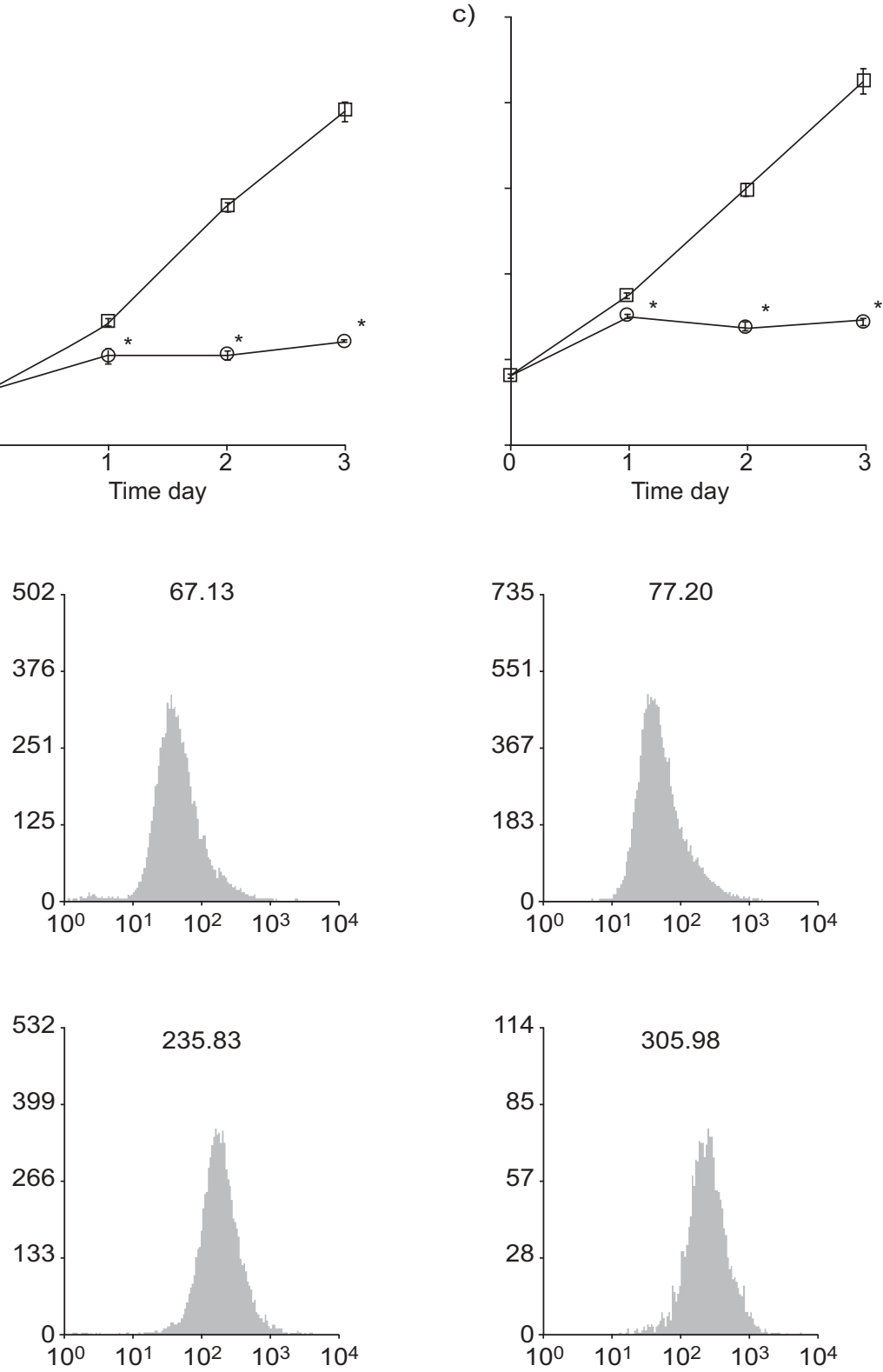

CFSE-log

FIGURE 6. Suberoylanilide hydroxamic acid (SAHA) inhibits fibroblast proliferation. a) Fetal lung fibroblasts (FLF), b) adult lung fibroblasts (ALF) and c) idiopathic pulmonary fibrosis lung fibroblasts (ILF) were cultured in $10 \%$ fetal bovine serum (FBS) with ( $\bigcirc$ ) or without ( $\square$ ) SAHA at $5 \mu$ M. Averages of cell numbers from triplicates were quantified by adherent cytometry at each time-point up to 3 days. *: $p<0.05$ compared with fibroblasts cultured in 10\% FBS at the same time-point. d) Changes of carboxyfluoroscein succinimidyl ester (CFSE) fluorescence intensity after 3 days without SAHA (top row) or with $5 \mu$ M SAHA (bottom row).

compound inhibited the transdifferentiation of fibroblasts to myofibroblasts, the cell type primarily responsible for pathological matrix accumulation. We also investigated the potential fibrolytic properties of SAHA and tested fibroblast collagenase, MMP1 and its inhibitor TIMP1, components responsible for remodeling of extracellular matrix. Surprisingly, the total potentially activated-MMP1 was doubled by TGF- $\beta 1$ in ALF and ILF. SAHA reversed it to the basal level. SAHA has been reported to inhibit TGF- $\beta 1$-induced TIMP1 expression in mouse fibroblast [16]. However, in our hands SAHA had no effect on TGF- $\beta 1$-induced TIMP1 production in human normal and ILF, and it even induced a further increase of TIMP1 in FLF. Therefore, it is conceivable that the net reduction of collagen deposition might be accredited to the effects of SAHA on collagen synthesis and secretion rather than collagen turnover. We have also shown the first evidence that SAHA inhibited fibroblast proliferation in serum.

SAHA is known as a broad-spectrum HDACi [17]. In comparison with published work on TSA, our data suggest 

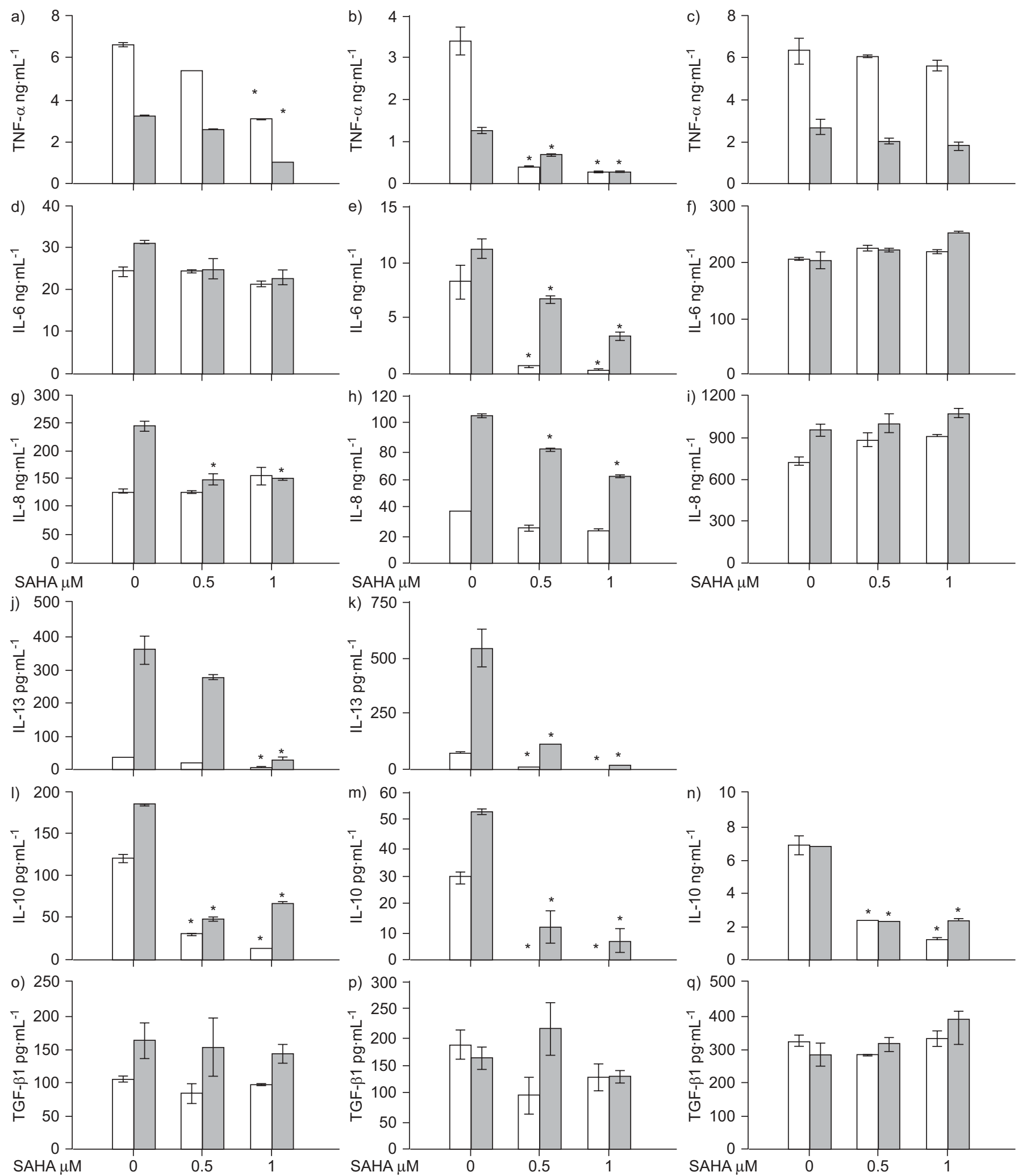

FIGURE 7. Suberoylanilide hydroxamic acid (SAHA) changes cytokine release in peripheral blood mononuclear cells (PBMC) and its subpopulations. a-c) tumour necrosis factor (TNF)- $\alpha, \mathrm{d}-\mathrm{f}$ ) interleukin (IL)-6, g-i) IL-8, j, k) IL-13, I-m) IL-10, and o-q) transforming growth factor (TGF)- $\beta 1$. SAHA at 0.5 or $1 \mu \mathrm{M}$ was added to PBMC (a, d, g, $\mathrm{j}, \mathrm{l}$, and o), lymphocytes (b, e, h, k, m and p) or peripheral monocytes (c, f, i, n and q) stimulated by $0.5 \mathrm{ng} \cdot \mathrm{mL}^{-1}$ phorbol myristate acetate plus $50 \mathrm{nM}$ calcium ionophore for $24 \mathrm{~h}(\square)$ or $48 \mathrm{~h}(\square)$. The experiments were performed three times with cells from three different donors. The averages of duplicate measurements from one representative experiment are shown. *: $p<0.05$ compared with untreated control. 


\begin{tabular}{lcccc} 
TABLE 1 & $\begin{array}{l}\text { Suberoylanilide hydroxamic acid changes } \\
\text { secretion of cytokines from peripheral blood } \\
\text { mononuclear cells (PBMC) } \\
\text { and subpopulations }\end{array}$ \\
& & PBMC & Lymphocytes & $\begin{array}{c}\text { Peripheral } \\
\text { monocytes }\end{array}$ \\
\hline Th1 & TNF- $\alpha$ & $\downarrow$ & $\downarrow$ & $\rightarrow$ \\
Pro-inflammatory & IL-6 & $\rightarrow$ & $\downarrow$ & $\rightarrow$ \\
Chemokine & IL-8 & $\downarrow$ & $\downarrow$ & $\rightarrow$ \\
Th2 & IL-13 & $\downarrow$ & $\downarrow$ & ND \\
& IL-10 & $\downarrow$ & $\downarrow$ & $\downarrow$ \\
Others & TGF- $\beta 1$ & $\rightarrow$ & $\rightarrow$ & $\rightarrow$ \\
\hline
\end{tabular}

Th; T helper; TNF: tumour necrosis factor; IL; interleukin; TGF: transforming growth factor; ND: not detected.

that inhibiting HDAC activity by SAHA interferes with the pro-fibrotic effects of TGF- $\beta 1$. Although Smad expression and activation are not significantly altered in the presence of TSA, TGF- $\beta 1$-induced expression of Sp1, an essential transcription factor of Smad-dependent collagen synthesis, is inhibited by TSA in foreskin fibroblasts [18]. Another line of evidence is that HDAC4, and to a lesser extent HDAC6 and HDAC8, are required for TGF- $\beta 1$-induced myofibroblastic differentiation. In particular, the expression of the endogenous repressor of the TGF- $\beta 1$ pathway, $5^{\prime}$-TG-3'-interacting factor, is stimulated by silencing HDAC4 [19]. As further evidence for the broadspectrum effects of SAHA, we observed hyperacetylated $\alpha$ tubulin in human lung fibroblasts in response to SAHA. This strongly indicates that HDAC6, a microtubule-associated deacetylase [20], is inhibited. Of note, fibroblast motility is strongly compromised with HDAC6 inhibition [21]. Therefore, (cross)inhibition of HDAC6 with SAHA might have an additional beneficial effect by inhibiting the invasive and contractile motility of myofibroblasts.

It is well known that inflammation typically precedes fibrosis in fibroproliferative diseases [22]. However, the role of inflammation in the early pathogenic stage of IPF is under debate because current anti-inflammatory therapy seems to be ineffective in IPF, yet patchy chronic interstitial inflammation is observed in the pathology of IPF [5]. Therefore, we also investigated the anti-inflammatory potential of SAHA. As the concentrations applied in fibroblast cultures caused apoptosis in PBMC and its subpopulations, we lowered the concentrations by five to 10 times, which is also in accordance with an earlier study [23]. A reduced secretion of cytokines was observed in PMA/CI activated lymphocytes and PBMC. Our observation of reduced secretion of IL-13 from lymphocytes in response to SAHA might be significant, as IL-13 is known to play a dominant role in pulmonary fibrosis. Overexpression of IL-13 in the lung generates severe subepithelial airway fibrosis [24]. IL-13 induces the production of TGF- $\beta 1$ in macrophages and also indirectly activates latent TGF- $\beta 1$ by stimulating MMP production [25]. Furthermore, IL-13 increases the expression of $\alpha$-SMA and collagen III in human primary lung fibroblasts, and induces apoptosis in lung epithelia or epithelial cell lines [26]. We also found that IL-10 was reduced in PBMC and its subpopulations. The exact role of this cytokine in pulmonary fibrosis remains to be established. Although delivery of IL-10 plasmid inhibits bleomycininduced pulmonary fibrosis [27], transgenic mice over expressing IL-10 develop pulmonary inflammation and subepithelial fibrosis with an accumulation of TGF- $\beta 1$ [28]. In accordance, IL-10 augments the fibrotic responses to inhaled silica particles as seen in IL-10 deficient mice [29] and over expression of IL-10 by adenoviral gene transfer [30]. In vitro, IL-10 also induces TGF- $\beta 1$ expression in alveolar macrophages [29]. Hence, inhibition of IL-13 and IL-10 by SAHA may have additional beneficial effects in IPF.

Of note, this is the first study evaluating a potential antifibrotic drug under conditions of enhanced matrix formation induced by macromolecular crowding [14]. We believe that such a biophysical culture environment is better suited than standard conditions in generating a comprehensive and functional extracellular matrix in a short-time frame, with all biochemical processes in place that represent fibrogenesis and fibrolysis [14]. This should add further weight to the conclusion derived herein, that SAHA possesses antifibrotic properties and is worth further exploration for a therapeutic option for pulmonary fibrosis.

\section{SUPPORT STATEMENT}

M. Raghunath recieved funding by a start-up grant from Provost and the Office of Life Sciences of National University of Singapore (R-397000-604-101; R-397-000-604-712), the Faculty of Engineering (R-397-000017-112) and the National Medical Research Council (R397-000-018213).

\section{STATEMENT OF INTEREST}

A statement of interest for M. Jung can be found at www.erj. ersjournals.com $/ \mathrm{misc} /$ statements.dtl

\section{ACKNOWLEDGEMENTS}

The authors would like to thank G. Lin (Immunology Program, National University of Singapore, Singapore) for expert technical help in flow cytometry and the National University of Singapore Tissue Engineering Program for strong support.

\section{REFERENCES}

1 American Thoracic Society/European Respiratory Society International Multidisciplinary Consensus Classification of the Idiopathic Interstitial Pneumonia. Am J Respir Crit Care Med 2002; 165: 277-304.

2 Scotton CJ, Chambers RC. Molecular targets in pulmonary fibrosis: the myofibroblast in focus. Chest 2007; 132: 1311-1321.

3 Gauldie J. Pro: Inflammatory mechanisms are a minor component of the pathogenesis of idiopathic pulmonary fibrosis. Am J Respir Crit Care Med 2002; 165: 1205-1206.

4 Strieter RM. Con: Inflammatory mechanisms are not a minor component of the pathogenesis of idiopathic pulmonary fibrosis. Am J Respir Crit Care Med 2002; 165: 1206-1207.

5 Maher TM, Wells AU, Laurent GJ. Idiopathic pulmonary fibrosis: multiple causes and multiple mechanisms? Eur Respir J 2007; 30: 835-839.

6 Bhatt N, Baran CP, Allen J, et al. Promising pharmacologic innovations in treating pulmonary fibrosis. Curr Opin Pharmacol 2006; 6: 284-292.

7 Bolden JE, Peart MJ, Johnstone RW. Anticancer activities of histone deacetylase inhibitors. Nat Rev Drug Discov 2006; 5: 769-784. 
8 Rishikof DC, Ricupero DA, Liu H, et al. Phenylbutyrate decreases type I collagen production in human lung fibroblasts. J Cell Biochem 2004; 91: 740-748.

9 Niki T, Rombouts K, De Bleser P, et al. A histone deacetylase inhibitor, trichostatin A, suppresses myofibroblastic differentiation of rat hepatic stellate cells in primary culture. Hematology 1999; 29: 858-867.

10 Rombouts K, Niki T, Greenwel P, et al. Trichostatin A, a histone deacetylase inhibitor, suppresses collagen synthesis and prevents TGF- $\beta(1)$-induced fibrogenesis in skin fibroblasts. Exp Cell Res 2002; 278: 184-197.

11 Huber LC, Distler JH, Moritz F, et al. Trichostatin A prevents the accumulation of extracellular matrix in a mouse model of bleomycin-induced skin fibrosis. Arthritis Rheum 2007; 56: 2755-2764.

12 Marks PA, Breslow R. Dimethyl sulfoxide to vorinostat: development of this histone deacetylase inhibitor as an anticancer drug. Nat Biotechnol 2007; 25: 84-90.

13 Stowell JC, Huot RI, Van Voast L. The synthesis of N-hydroxy-N phenyloctanediamide and its inhibitory effect on proliferation of AXC rat prostate cancer cells. J Med Chem 1995; 38: 1411-1413.

14 Lareu RR, Subramhanya KH, Peng Y, et al. Collagen matrix deposition is dramatically enhanced in vitro when crowded with charged macromolecules: the biological relevance of the excluded volume effect. FEBS Lett 2007; 581: 2709-2714.

15 Gribbin J, Hubbard RB, Le Jeune I, et al. Incidence and mortality of idiopathic pulmonary fibrosis and sarcoidosis in the UK. Thorax 2006; 61: 980-985.

16 Young DA, Billingham O, Sampieri CL, et al. Differential effects of histone deacetylase inhibitors on phorbol ester- and TGF- $\beta 1$ induced murine tissue inhibitor of metalloproteinases-1 gene expression. FEBS J 2005; 272: 1912-1926.

17 Arts J, Angibaud P, Mariën A, et al. R306465 is a novel potent inhibitor of class I histone deacetylases with broad-spectrum antitumoral activity against solid and haematological malignancies. Br J Cancer 2007; 97: 1344-1353.

18 Ghosh AK, Mori Y, Dowling E, et al. Trichostatin A blocks TGF- $\beta$ induced collagen gene expression in skin fibroblasts: involvement of Sp1. Biochem Biophys Res Commun 2007; 354: 420-426.
19 Glenisson W, Castronovo V, Waltregny D. Histone deacetylase 4 is required for TGF $\beta 1$-induced myofibroblastic differentiation. Biochim Biophys Acta 2007; 1773: 1572-1582.

20 Hubbert C, Guardiola A, Shao R, et al. HDAC6 is a microtubuleassociated deacetylase. Nature 2002; 417: 455-458.

21 Tran AD, Marmo TP, Salam AA, et al. HDAC6 deacetylation of tubulin modulates dynamics of cellular adhesions. J Cell Sci 2007; 120: 1469-1479.

22 Wynn TA. Common and unique mechanisms regulate fibrosis in various fibroproliferative diseases. J Clin Invest 2007; 117: 524-529.

23 Bode KA, Schroder K, Hume DA, et al. Histone deacetylase inhibitors decrease Toll-like receptor-mediated activation of proinflammatory gene expression by impairing transcription factor recruitment. Immunology 2007; 122: 596-606.

24 Zhu Z, Homer RJ, Wang Z, et al. Pulmonary expression of interleukin-13 causes inflammation, mucus hypersecretion, subepithelial fibrosis, physiologic abnormalities, and eotaxin production. J Clin Invest 1999; 103: 779-788.

25 Lanone $\mathrm{S}$, Zheng $\mathrm{T}$, Zhu Z, et al. Overlapping and enzyme-specific contributions of matrix metalloproteinases-9 and -12 in IL-13induced inflammation and remodelling. J Clin Invest 2002; 110: 463-474.

26 Borowski A, Kuepper M, Horn U, et al. Interleukin-13 acts as an apoptotic effector on lung epithelial cells and induces pro-fibrotic gene expression in lung fibroblasts. Clin Exp Allergy 2008; 38: $619-628$.

27 Nakagome K, Dohi M, Okunishi K, et al. In vivo IL-10 gene delivery attenuates bleomycin induced pulmonary fibrosis by inhibiting the production and activation of TGF- $\beta$ in the lung. Thorax 2006; 61: 886-894.

28 Lee CG, Homer RJ, Cohn L, et al. Transgenic overexpression of interleukin (IL)-10 in the lung causes mucus metaplasia, tissue inflammation, and airway remodeling via IL-13-dependent and -independent pathways. J Biol Chem 2002; 277: 35466-35474.

29 Barbarin V, Arras M, Misson P, et al. Characterization of the effect of interleukin-10 on silica-induced lung fibrosis in mice. Am J Respir Cell Mol Biol 2004; 31: 78-85.

30 Barbarin V, Xing Z, Delos M, et al. Pulmonary overexpression of IL-10 augments lung fibrosis and Th2 responses induced by silica particles. Am J Physiol Lung Cell Mol Physiol 2005; 288: L841-L848. 